\title{
The Evolution and Performance of the ATLAS Calorimeter-Based Triggers in 2011 and 2012
}

\author{
IEEE Real Time 2012
}

12 June 2012

Ivana Hristova (HUB) on behalf of the ATLAS collaboration

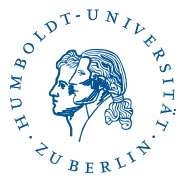




\section{Introduction}

- CERN Large Hadron Collider

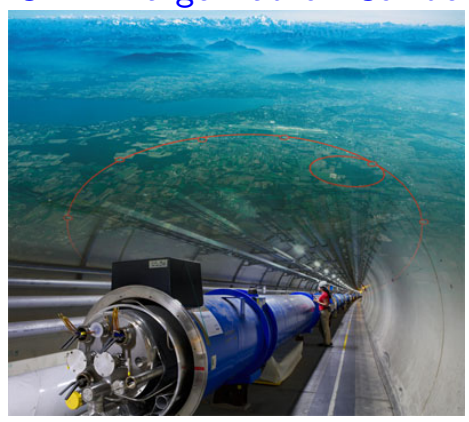

- Trigger Signatures

- Electron and photon: EM

- Tau ( $\tau$-leptons): TAU

- Jet: J

- Total $\left(\sum E_{\mathrm{T}}\right)$ and Missing $\left(E_{\mathrm{T}}^{\text {miss }}\right)$ Transverse Energy $\left(E_{T}\right)$ : TE and $X E$

- XE-Significance: $X S \sim \frac{X E}{\sqrt{T E}}$
- ATLAS calorimeter

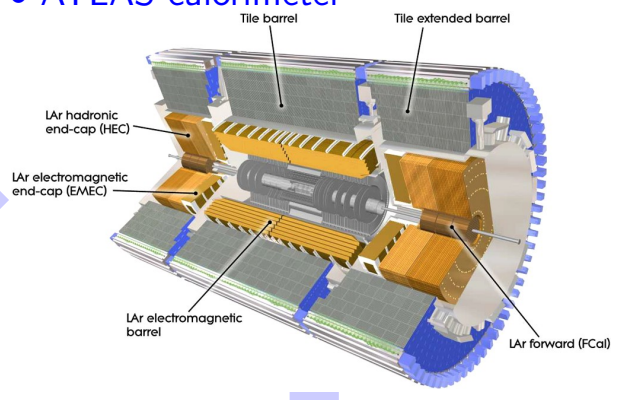

- ATLAS Trigger input rate $\leq 40 \mathrm{MHz}$ \begin{tabular}{l|ll}
\hline event selection & time out. rate
\end{tabular} \begin{tabular}{l|ll}
\hline Level-1 (L1) & $<2.5 \mu \mathrm{s}<75 \mathrm{kHz}$
\end{tabular} Level-2 (L2) $\quad \sim 60 \mathrm{~ms} \sim 5 \mathrm{kHz}$ Event Filter (EF) $\quad \sim 1 \mathrm{~s} \quad \sim 0.4 \mathrm{kHz}$ L1 (hardware) and HLT (software) High Level Trigger (HLT): L2 and EF 


\section{LHC Luminosity Evolution in 2011-2012}

- Change of beam parameters $\rightarrow$ steady increase of instantaneous luminosity and average number of interactions per bunch crossing (pile-up) $\rightarrow$ higher event rates $\rightarrow$ higher load on all trigger levels, rate=luminosity $\times$ cross section

\begin{tabular}{|c|c|c|c|c|}
\hline \multicolumn{2}{|c|}{ 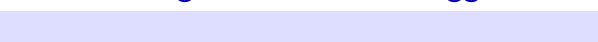 } & 2011 & 2012 & nominal \\
\hline & energy per beam [ $\mathrm{TeV}]$ & 3.5 & 4.0 & 7.0 \\
\hline & bunch crossing (BC) frequency [MHz] & 20 & 20 & 40 \\
\hline & bunch spacing $[\mathrm{ns}]$ & 50 & 50 & 25 \\
\hline & number of filled bunches & 1380 & 1380 & 2808 \\
\hline$\beta^{*}$ & amplitude function $[\mathrm{m}]$ & 1.0 & 0.6 & 0.55 \\
\hline$L_{\text {peak }}$ & peak luminosity $\left[10^{33} \mathrm{~cm}^{-2} \mathrm{~s}^{-1}\right]$ & 3.65 & $6.0(6.8)$ & 10 \\
\hline$\langle\mu\rangle$ & average number of interactions per $B C$ & 17 & $28(35)$ & 19 \\
\hline
\end{tabular}
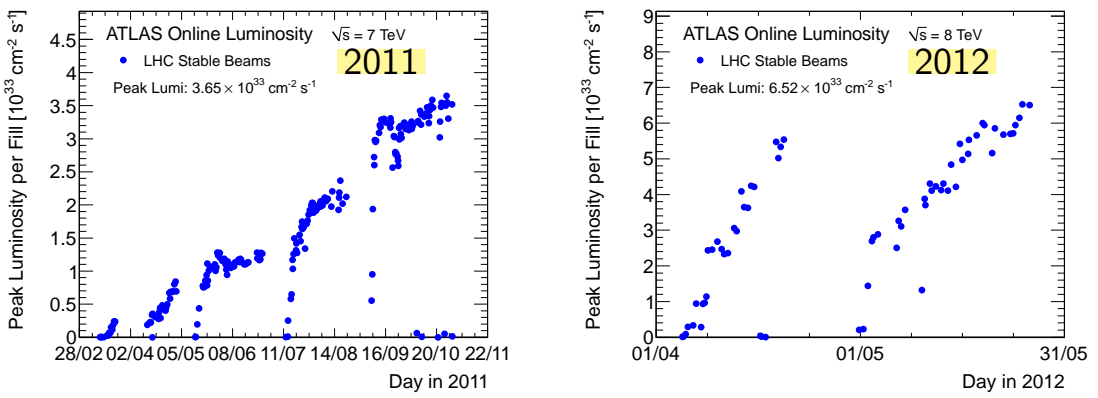

Day in 2011

Day in 2012 


\section{Level-1 Calorimeter Trigger (L1Calo)}

- Receives $~ 7200$ analog signals (trigger towes) from the ATLAS calorimeter

- Digitisation, $E_{\mathrm{T}}$ calibration ( $\mathrm{ADC} \rightarrow \mathrm{GeV}$, suppress signal if $E_{\mathrm{T}}<E_{\mathrm{T}, \text { thresh. }}$ )
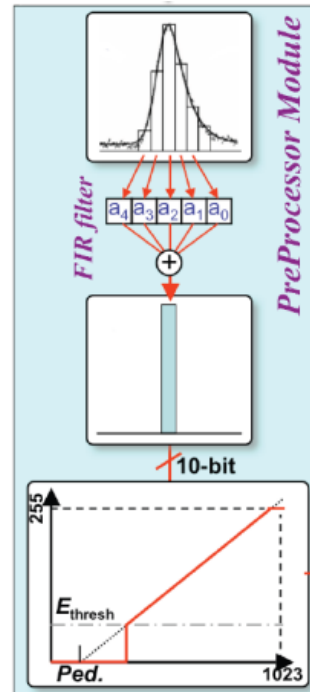

Trigger signature identification

- trigger towers $E_{\mathrm{T}}, \Delta \eta \times \Delta \phi$ sliding window algorithm

- identify regions of interest (Rols) with largest local $E_{\mathrm{T}}$

- count number of Rols above given $E_{\mathrm{T}}$ threshold, ie $E_{\mathrm{T}}>3 \mathrm{GeV}(\mathrm{EM} 3)$

- compute global quantities $\sum E_{\mathrm{T}}, E_{\mathrm{T}}^{\mathrm{miss}}, E_{\mathrm{T}}^{\text {miss }}$-sig.

- Rols locations in $(\eta, \phi)$ sent to L2

Jet algorithm:

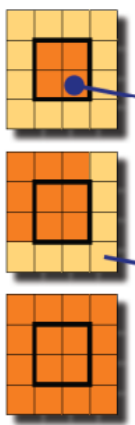

EM cluster algorithm:

$0.4 \times 0.4$

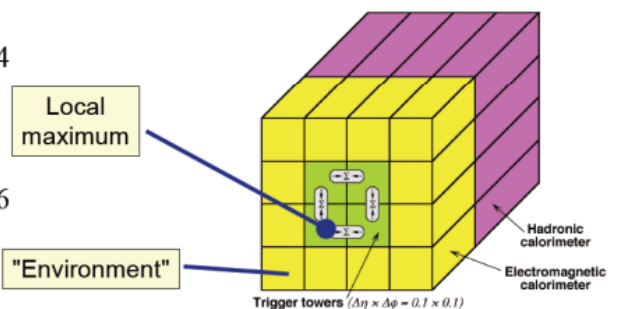

$0.8 \times 0.8$
Trigger towers $(\Delta \eta \times \Delta \phi-0.1 \times 0.1)$

(1) Vertical Sums

$\Theta-$ Horizontal Sums

t., De-cluster/Rol region:

to De-cluster/Rol regil maximum

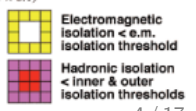




\section{L1Calo Forward Region Noise Suppression}

- Clear pile-up dependence in width, $\sigma$, of the ADC count (noise) distribution in the forward calorimeter region (FCAL)

- Pile-up noise width varies as a function $\eta$, e.g., FCAL regions in $\eta$ :

$$
\begin{array}{ll}
\text { bin } 1 & 3.1<|\eta|<3.2 \\
\text { bin 2 } & 3.2<|\eta|<3.5 \\
\text { bin 3 } & 3.5<|\eta|<4.2 \\
\text { bin 4 } & 4.2<|\eta|<4.9
\end{array}
$$
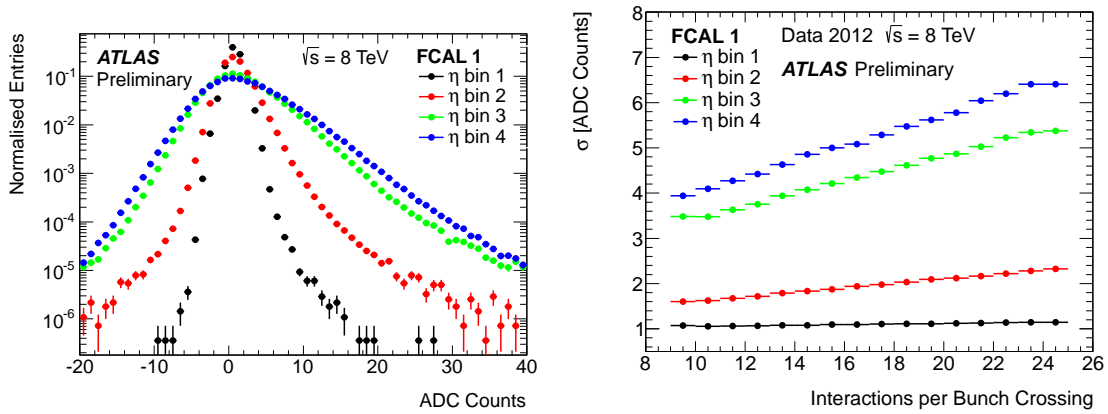


\section{L1Calo Forward Region Noise Suppression}

- Pile-up noise from FCAL dominated $E_{\mathrm{T}}^{\text {miss }}$ trigger rate in 2011

- $E_{\mathrm{T}}^{\text {miss }}$ trigger thresholds increased several times in 2011

- Raised noise cuts in FCAL region to strongly suppress rate

- Have checked this has minimal impact on trigger efficiency

- This allowed thresholds to be lowered at L1 $\rightarrow$ great benefit for physics analysis
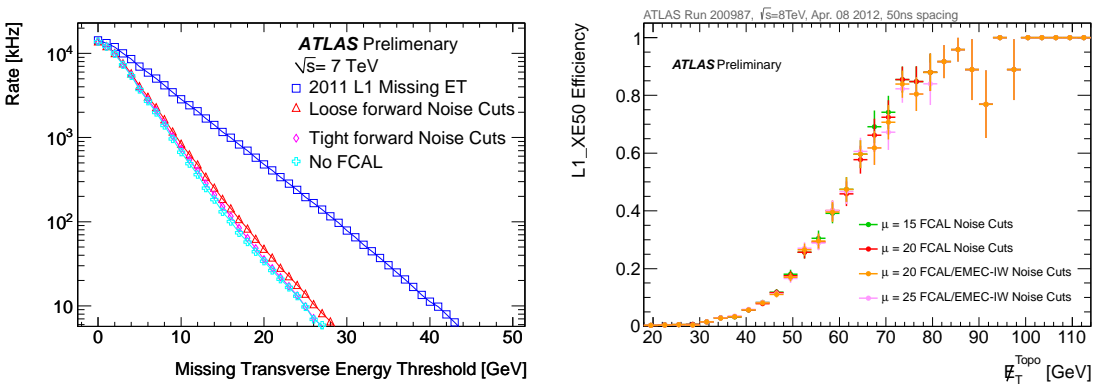


\section{L1Calo-Based Trigger Signatures}

- Need to provide robust selection and keep rates under control as the average number of interactions per bunch crossing (pile-up) increases

- Understanding how current rates behave allows us to predict them at different LHC conditions

- Single-object rates: TAU15, J75, EM30

- Almost pile-up independent

- Multi-object rates: 4J10

- Non-linear dependence expected and can be explained on statistical basis

- FJ75, L1_XE50

Exponential rise caused by higher occupancy in the forward calorimeter region supported by the larger trigger tower sizes

- Non-linear rates controlled by increase of pile-up noise cuts in the forward calorimeter region (previous page)

- XE50_BGRP7: removed triggering on the first few bunches per train where calorimeter response is elevated (out-of-time pile-up)

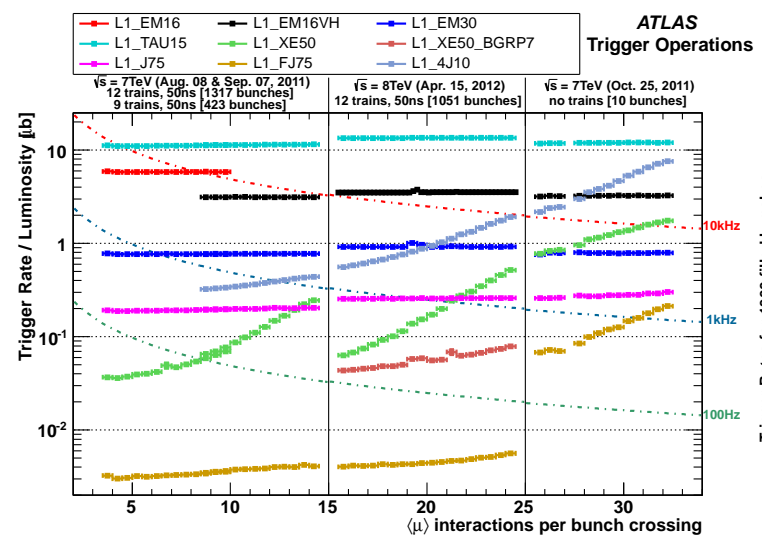




\section{Electron Trigger Signature: Evolution and Performance}

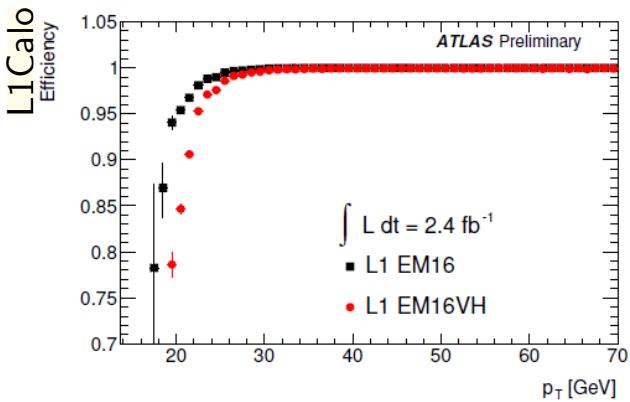

- Adjust thresholds to fit within rate budget

- Tightened ( $\mathrm{T}) \mathrm{L} 1$ selection

- EM16 $\rightarrow$ EM16VH $\rightarrow$ EM18VH

$-2 \mathrm{EM} 7 \rightarrow 2 \mathrm{EM} 10 \rightarrow 2 \mathrm{EM} 10 \mathrm{VH}$

- $E_{\mathrm{T}}$ threshold varied (V) vs $\eta$ and $E_{\mathrm{T}}<1 \mathrm{GeV}$ in hadron $(\mathrm{H})$ isolation ring, achieved $50 \%$ rate reduction compared to EM16

- Raised HLT threshold $20 \rightarrow 22 \rightarrow 24 \mathrm{GeV}$

- Tighter shower shape ID, medium $\rightarrow$ medium1

- Electron efficiencies remain high $(Z \rightarrow e e$ tag-and-probe method used)
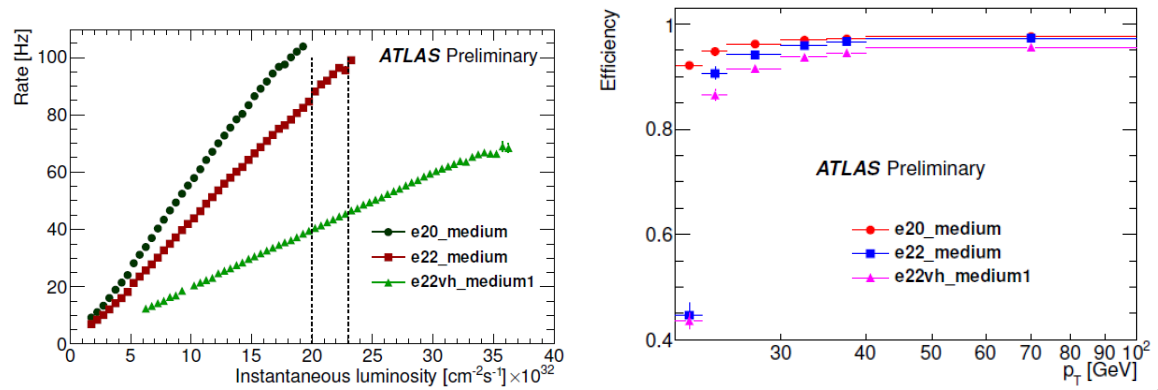


\section{Photon Trigger Signature: Rates and Performance}

- Photon triggers serve a broad physics programme

- Standard Model $\gamma$-production cross section, the Higgs search channel $H \rightarrow \gamma \gamma$, searches of physics beyond the SM

- Stable rates and high efficiencies throughout 2011 and 2012

- Rapid turn on curves, no significant dependence on $\eta$

. No strong dependence on pile-up (number of reconstructed primary vertices)
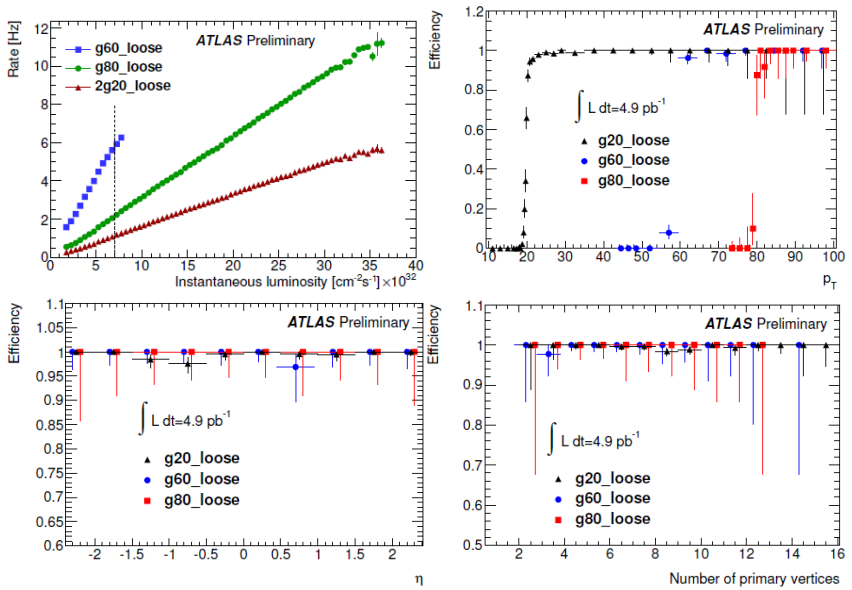


\section{Tau Trigger Signature: Rates}

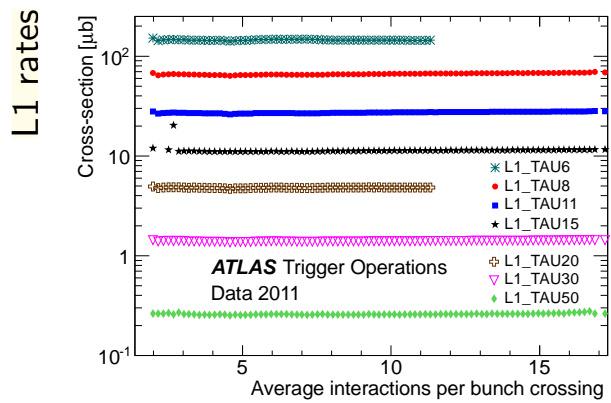

- L1 rates well behaved

- L1 trigger rates scale linearly with luminosity

- 2012: moderate increase of $E_{\mathrm{T}}$ thresholds, EM isolation (I) $<5 \mathrm{GeV}$

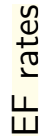

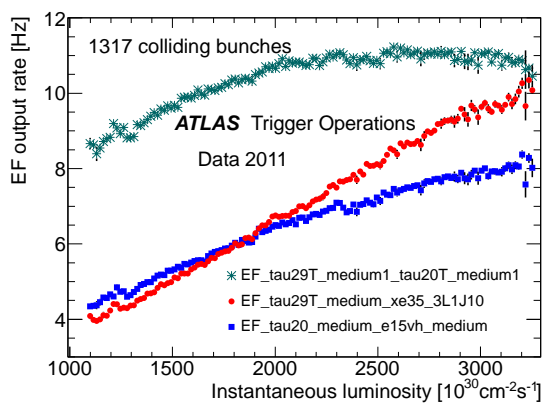

- $E_{\mathrm{T}}$ in EM-cone of size 0.4 , cone size very sensitive to pile-up

- EF single and di-tau triggers saturate at high luminosity

- Combined triggers rise non-linearly with luminosity

- Non-linear rates may lead to loss of efficiencies 


\section{Tau Trigger Signature: Various Improvements}

- Move to pile-up robust variables

- Smaller cone sizes: from 0.4 to 0.2

- $\Delta Z$ cut in track-based parameters

- More offline-like variables used

- EF now uses multivariate selection

- Boosted decision tree (BDT)

- Log-likelihood (LLH)

Good example of moving offline selection into the trigger to maintain high efficiency at low rate

\section{- 2011 data: cut based selection at EF}

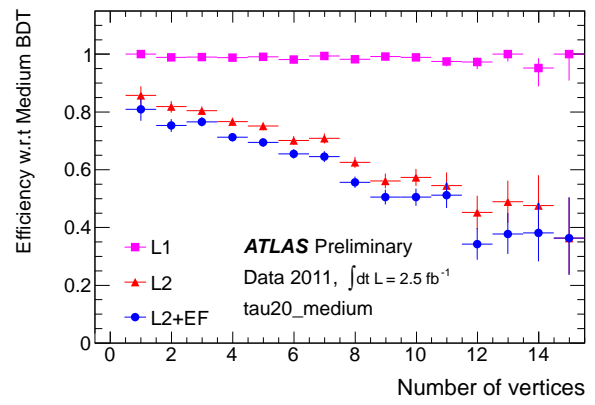

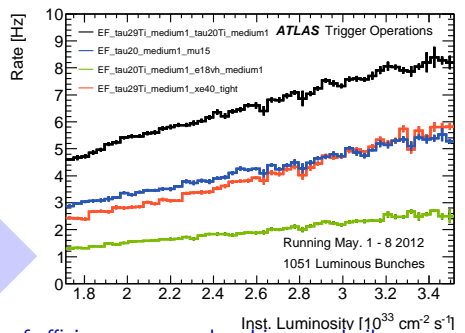

- Loss of efficiency recovered and improved pile-up dependence, 2011 data reprocessed with 2012 algorithms:

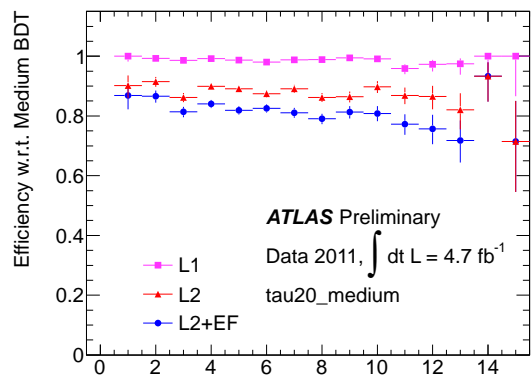

Number of vertices 


\section{Jet Trigger Signature: 2011}

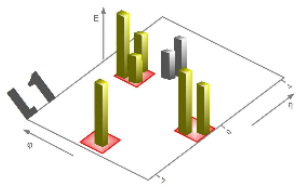

- Rol approach, limited performance as pile-up increased

- Poor resolution, efficiency loss for close-by jets in multi-jet events

- L1: $(0.2 \times 0.2)$ jet elements and $4 \times 4$ JE sliding window

- L2: Jet finding using calorimeter cells in L1 Rol cone

- EF: Full calorimeter

Clusters from all calorimeter cell, anti- $k_{\mathrm{T}}$ algorithm using clusters

- Improved position resolution

- Increased acceptance for wide jets unseeded jet finding with sliding windows using L1Calo towers

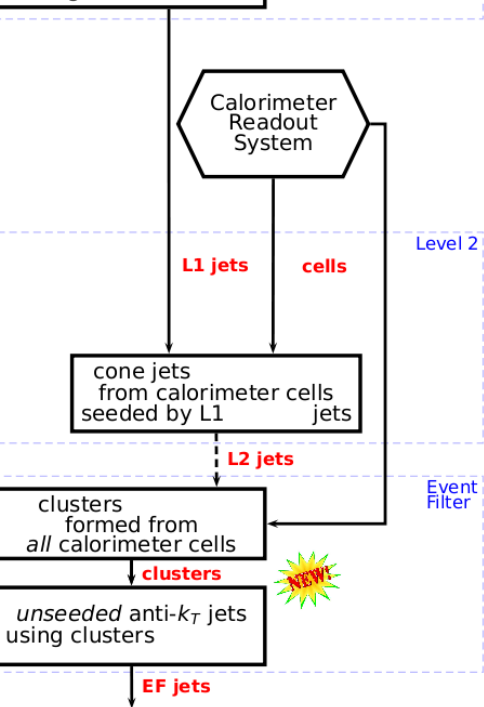

Level 1
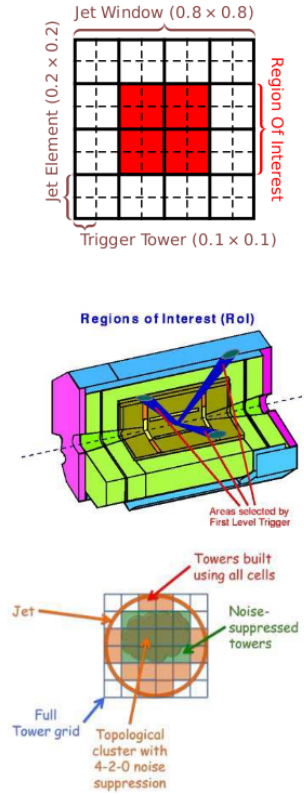


\section{Jet Trigger Signature: 2012}

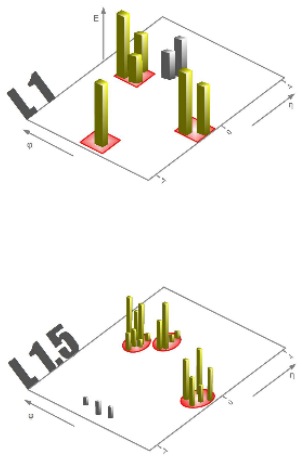

- New: L1.5 trigger

- Allows to run anti- $k_{\mathrm{T}}$, etc. algorithms at L2 using all 1Calo trigger towers

Better resolution, improved L1 efficiency for multi-jet events

- New at L2: anti- $k_{\mathrm{T}}$ jets from cells seeded by $L 1.5$ jets

- New at EF: towers using all calorimeter cells

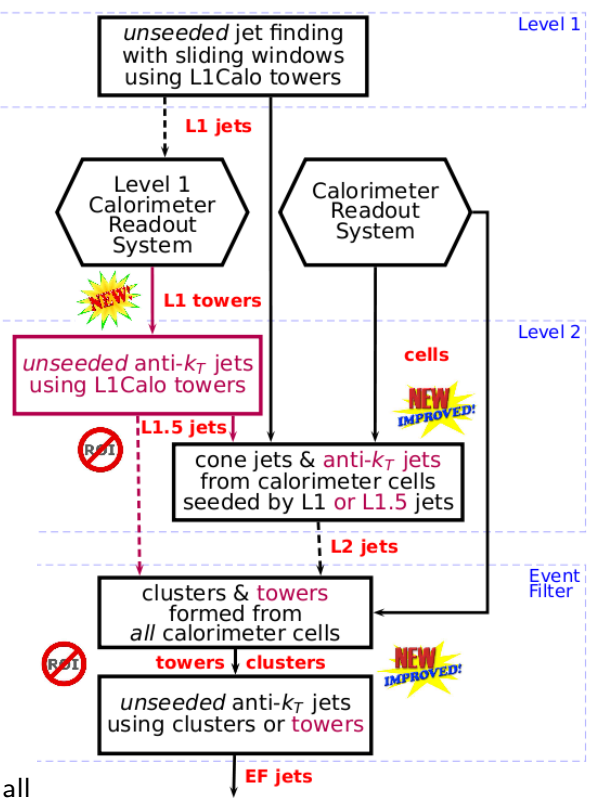

unseeded jet finding

with sliding windows

using L1Calo towers

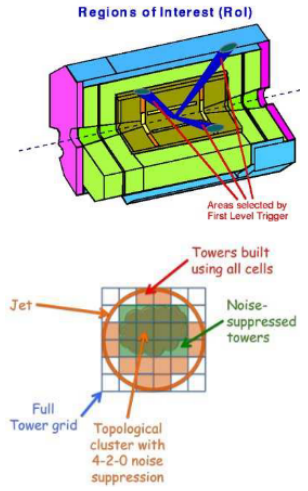

$13 / 17$ 


\section{Jet Trigger Signature: Performance}

- L1.5 jet finding within the L2 timing requirements
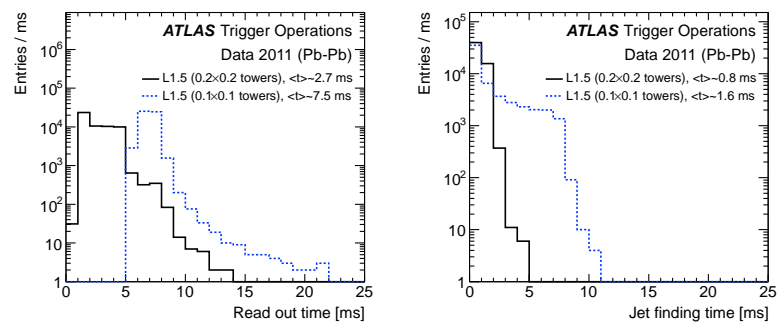

- Readout full L1Calo trigger-tower information

- Run offline-like algorithms using trigger towers

- Tested in $\mathrm{Pb}-\mathrm{Pb}$ collisions in 2011

- Optimised in 2012 for increased pile-up conditions

- Improved resolution and efficiency (due to anti- $k_{\mathrm{T}}$ )
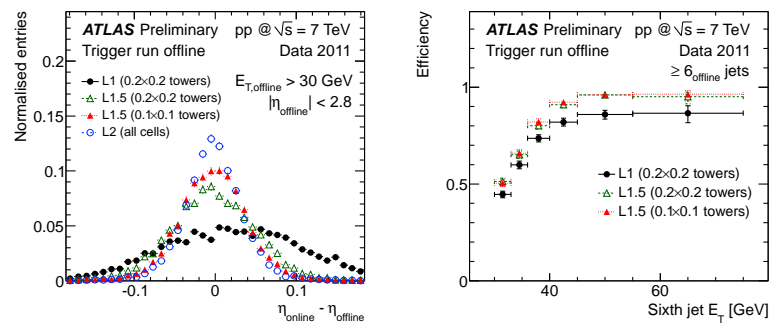

- anti- $k_{\mathrm{T}}$ (L1.5) more precise than sliding window (L1) clusters

- Resolution improved for jets at low $E_{\mathrm{T}}$

- Efficiency recovered for events with $\geq 6$ jets 


\section{$E_{\mathrm{T}}^{\text {miss }}$ Trigger Signature: Evolution}

- Trigger on global quantitites: $\sum E_{\mathrm{T}}, E_{\mathrm{T}}^{\mathrm{miss}}, \frac{E_{\mathrm{T}}^{\mathrm{miss}}}{\sqrt{\sum E_{\mathrm{T}}}}$

- Sensitivity to detector resolution (noise, pile-up) at low $E_{\mathrm{T}}^{\text {miss }} \rightarrow$ rates to depend non-linearly on instantaneous luminosity and $\mu \rightarrow$ model needed to predict rates at various LHC conditions

- Evolution in 2011 and 2012

- 2011: L1 output = EF input rate as only Rols available at L2, thresholds raised

- 2012: At L2, access to digitised signals from all calorimeter cells using calorimeter readout

- Resolution improves by about $50 \%$, rejection rate improved by a factor of 5-6, trigger thresholds lowered

- 2012: At EF, topological cluster algorithms use local weight calibration

- No BCID dependent cell energy corrections yet, significant gain in resolution, processing time (computing cost) pile-up dependent
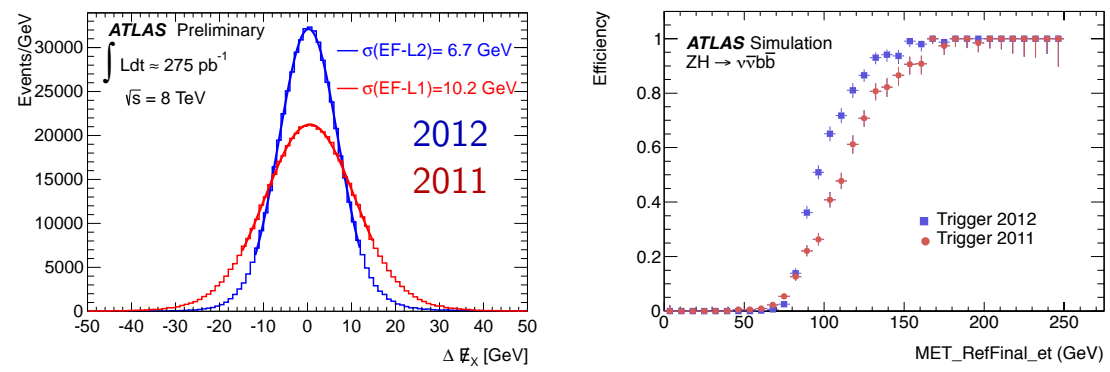


\section{Summary}

- In 2011 and 2012: The LHC parameters steadily increased, the luminosity from 0.1 to $\sim 6.7 \times 10^{33} \mathrm{~cm}^{-2} \mathrm{~s}^{-1}$ and the pile-up from 1 to $\sim 30$

- The ATLAS calorimeter-based trigger signatures represented about $2 / 3$ of the Level-1 trigger bandwidth of $\lesssim 60 \mathrm{kHz}$

- The flexibility of the Level-1 Calorimeter Trigger allowed various improvements to be implemented to cope with the evolving LHC conditions

- $\eta$-dependent noise-cut optimisation in the forward calorimeter region for high pile-up

- The software-based High Level Trigger continuously evolved

- Faster and advanced algorithms implemeted at trigger level to perform more offline-like selection, i.e., using larger amounts, finer-granularity and more precise readout information

- The performance of the trigger signatures was studied in detail to ensure rapid feedback and unbiased selection for physics analyses

- Electron and photon: increased thresholds to reduce rate, isolation/veto and tighter selection criteria used at negligible loss of efficiency

- Tau: various improvements based on the use of additional variables and improved selection techniques, loss of efficiencies recovered

- Jet: improved rates, efficiency and position resolution for multi-jet events and low $E_{\mathrm{T}}$ jets

- $E_{T}^{\text {miss }}$ : Understanding how rates depend on pile-up allows for more reliable rate predictions and the development of pile-up robust triggers 


\section{BACKUP $E_{T}^{\text {miss }}$ Trigger Signature: Significance}
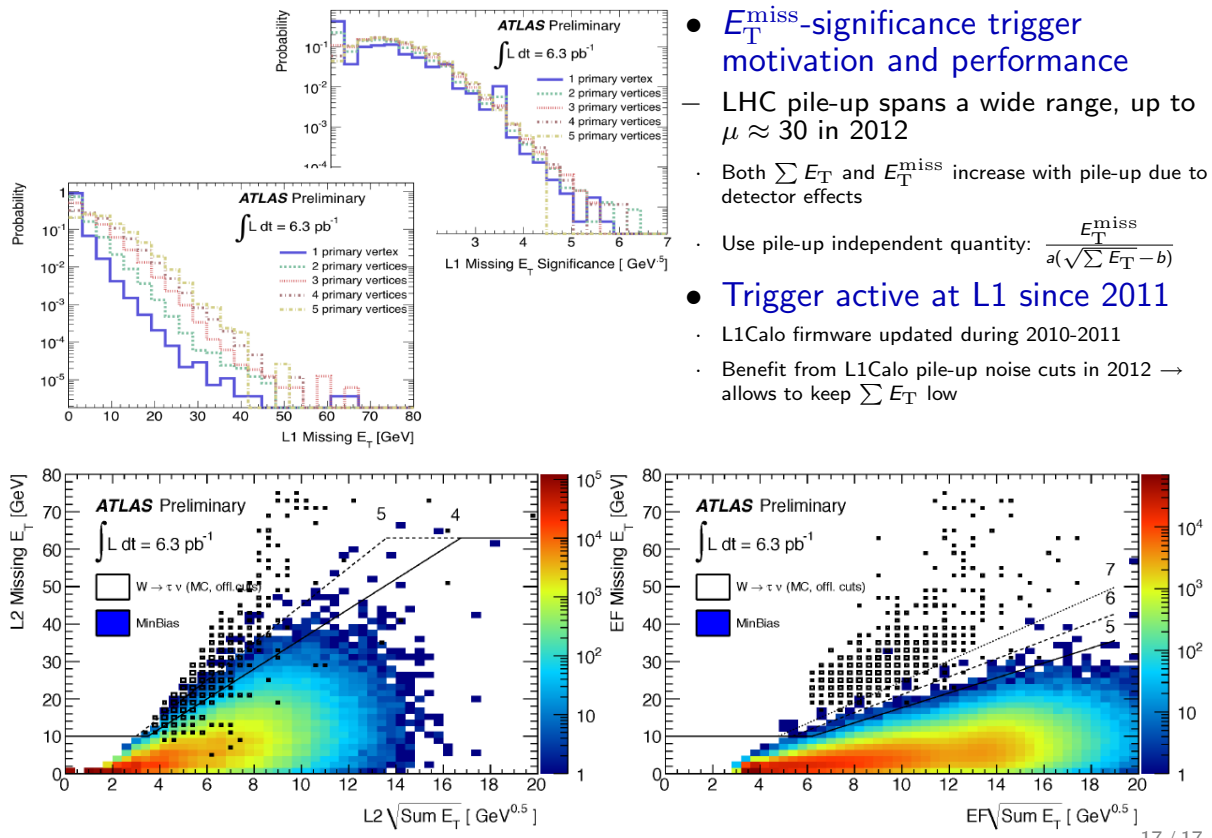